\title{
O EFEITO DE DIFERENTES NÍVEIS DE ATIVIDADE FÍSICA NA FUNÇÃO COGNITIVA E DEPRESSÃO EM IDOSOS BRASILEIROS
}

THE EFFECT OF DIFFERENT LEVELS OF PHYSICAL ACTIVITY IN THE COGNITIVE FUNCTION AND DEPRESSION IN BRAZILIANS ELDERLY

EL EFECTO DE DIFERENTES NIVELES DE ACTIVIDAD FÍSICA EN LA FUNCIÓN COGNITIVA Y DEPRESIÓN EN ANCIANOS BRASILEÑOS

\author{
Edivana Almeida*(edivana_aguiar@yahoo.com.br)* \\ Isabel Mourão**(mimc@utad.com)* \\ Eduarda Coelho***(ecoelho@utad.pt)*
}

\section{RESUMO}

O objetivo deste estudo foi verificar o efeito de diferentes níveis de atividade física na função cognitiva e depressão em idosos. Método: Participaram 465 idosos brasileiros voluntários de ambos os sexos, praticantes e não praticantes de diferentes programas de atividade física (69,93 \pm 6,63 anos), divididos em 4 grupos conforme critérios do International Physical Activity Questionnaire (IPAQ): idosos ativos, insuficientemente ativos $\mathrm{A}$, insuficientemente ativos $\mathrm{B}$ e sedentários. Para verificar possíveis declínios da função cognitiva, utilizou-se o Mini Exame do Estado Mental (MEEM) e para verificar a intensidade da depressão, o Inventário de Depressão de Beck (BDI-II). A recolha dos dados ocorreu em 3 momentos avaliativos distintos (avaliação inicial, avaliação intermédia e avaliação final) ao longo de 35 semanas. Resultados: Para a função cognitiva foi encontrado um efeito significativo dos diferentes níveis de atividade física na avaliação intermédia e na avaliação final $(p=0.000)$. Para a depressão, os resultados indicaram um efeito significativo dos diferentes níveis de atividade física nos 3 momentos avaliativos ( $p=0.000$ ). Os resultados nos permitem concluir que a prática regular de atividade física moderada demonstrou melhorias na função cognitiva e depressão de idosos brasileiros.

Palavras-chave: Idosos; Função Cognitiva; Depressão; Níveis de atividade físicas 


\title{
Egitania
}

s c i e $\Omega$ c i a

\begin{abstract}
The objective of this study was to verify the effect of different levels of physical activity on cognitive function and depression in the elderly. Method: Participants were 465 Brazilian elderly volunteers, some practicing and others not practicing different physical activity programs (69.93 \pm 6.63 years), divided into 4 groups: active elderly, insufficiently active A, insufficiently active B and sedentary elderly, according to the criteria of the International Physical Activity Questionnaire (IPAQ). The Mini Mental State Examination (MMSE) was used to verify possible declines in cognitive function, and the Beck Depression Inventory (BDI-II) was used to verify the intensity of depression. The data collection took place in 3 distinct evaluative moments (initial, intermediate and final evaluation) over 35 weeks. Results: For cognitive function, a significant effect of the different levels of physical activity was found in the intermediate evaluation and in the final evaluation $(\mathrm{p}=0.000)$. For depression, the results indicated a significant effect of the different levels of physical activity in the three evaluative moments $(p=0.000)$. The results allow us to conclude that the regular practice of moderate physical activity demonstrated improvements in the cognitive function and depression of the Brazilian elderly.
\end{abstract}

keywords: Elderly; Cognitive Function; Depression; Levels of physical activity.

\section{RESUMEN}

El objetivo de este estudio fue verificar el efecto de diferentes niveles de actividad física en la función cognitiva y depresión en ancianos. Método: Participaron 465 ancianos brasileños voluntarios, practicantes y no practicantes de diferentes programas de actividad física $(69,93 \pm 6,63$ años) divididos en 4 grupos: ancianos activos, insuficientemente activos $A$, insuficientemente activos B y sedentarios, conforme los criterios del International Physical Activity Questionnaire (IPAQ). Se utilizó el Mini Mental State Examination (MMSE) para verificar posibles declinaciones de la función cognitiva, y el Inventario de Depresión de Beck (BDI-II) para comprobar la intensidad de la depresión. La recogida de los datos ocurrió en 3 momentos de evaluación distintos (evaluación inicial, evaluación intermedia y evaluación final) a lo largo de 35 semanas. Resultados: Para la función cognitiva, se encontró un efecto significativo de los diferentes niveles de actividad física en la evaluación intermedia y en la 


\section{Egitania}

s c i e $n$ c i a

evaluación final ( $p=0.000)$. Para la depresión, los resultados indicaron un efecto significativo de los diferentes niveles de actividad física en los tres momentos de evaluación ( $\mathrm{p}=0.000$ ). Los resultados nos permiten concluir que la práctica regular de actividad física moderada demostró mejoras en la función cognitiva y depresión de los ancianos brasileños.

Palabras clave: Ancianos; Función Cognitiva; Depresión; Niveles de actividad física

*Edivana Almeida (PhD). Professora da Universidade Aberta a Terceira Idade (UATI) da Universidade do Estado da Bahia (UNEB). Psicóloga do Centro de Convivência do Idoso (CCl) do Hospital Naval de Salvador (HNSal) da Marinha do Brasil.

**Isabel Mourão (PhD). Professora Associada, aposentada do Departamento de Ciências do Desporto, Exercício e Saúde da Universidade de Trás-os-Montes e Alto Douro - UTAD. Investigadora do Centro de Investigação em Desporto, Saúde e Desenvolvimento Humano (CIDESD), UTAD, Vila Real, Portugal.

${ }^{* * *}$ Eduarda Coelho (PhD). Professora do Departamento de Ciências do Desporto, Exercício e Saúde da Universidade de Trás-os-Montes e Alto Douro - UTAD. Investigadora do Centro de Investigação em Desporto, Saúde e Desenvolvimento Humano (CIDESD), UTAD, Vila Real, Portugal. 


\section{INTRODUÇÃO}

A participação de idosos em programas de exercício físico é uma intervenção eficaz para manter ou melhorar o desempenho da função cognitiva e prevenir ou reduzir os níveis da depressão no envelhecimento (Benedetti, Borges, Petroski, \& Gonçalves, 2008). Os possíveis benefícios do exercício físico podem ser explicados por processos neurofisiológicos subjacentes ao efeito dos níveis superiores de atividade física na função cognitiva e na depressão no envelhecimento (Maass et al, 2016).

Estudos acerca dos processos neuropsicológicos indicam que a função cognitiva pode ser beneficiada pela prática regular da atividade física por promover o aumento da expressão de inúmeros fatores neurotróficos, especialmente do Brain Derived Neurotrophic Factor (BDNF), um dos fatores fundamentais ao desenvolvimento da neurogênese adulta em estruturas cerebrais fundamentais ao desempenho satisfatório da função cognitiva (Erickson et al, 2011) e por estimular a elevação do fluxo sanguíneo cerebral, que é mediada, principalmente pelo vascular endothelial growth factor (VEGF) e hormônios pluripotentes, como o insulin-like growth factor-1 (IGF-1), cujas atividades também foram associadas a angiogênese com repercussões benéficas à função cognitiva (Sonntag, Eckman, Ingraham \& Riddle, 2007; Maass et al., 2016).

A depressão, de acordo com a literatura, expressa o efeito da prática regular de atividade física ao demonstrar redução em seus níveis e até mesmo prevenção dos seus sintomas. Uma das hipóteses mais referenciadas é relativa ao aumento das monoaminas biogênicas cerebrais, principalmente da serotonina, noradrenalina e da dopamina (Frazer, Christensen \& Griffiths, 2005), cuja redução é considerada a causa direta da depressão, por serem fundamentais na regulação do comportamento direcionado à alguma meta e ao controle e adaptação do estresse (Krishnan \& Nestler, 2008).

Neste sentido, a depressão no idoso pode ter seus níveis reduzidos ou suprimidos com a prática regular de atividade física (Blumenthal, Smith \& Hoffman, 2012; Paranthaman et al, 2012), inclusive pode ser um complemento à terapia farmacológica no tratamento de idosos com o diagnóstico de depressão (Carneiro, Fonseca, Vieira-Coelho, Mora \& Vasconcelos-Raposo, 2015). 
São vários os estudos que revelam a ação benéfica da atividade física na cognição (Colcombe \& Kramer, 2003; Hötting \& Röder, 2013; WHO, 2010), especificamente, o contributo do exercício aeróbio, com benefícios ao nível do funcionamento cognitivo, especialmente na função executiva em pessoas idosas (Colcombe \& Kramer, 2003; Hilman et al., 2008), assim como, na modulação da depressão (Deslandes, 2013; Underwood et al., 2013).

Relativamente à relação entre intensidade do exercício e cognição os resultados evidenciam que os benefícios podem variar de acordo com o timing; a performance cognitiva parece ser beneficiada logo após quando o exercício físico é de baixa intensidade (efeito agudo), enquanto que uma elevada intensidade apresenta melhorias depois de passado algum tempo (efeito crônico) (Chang et. al., 2012).

Por isso, torna-se necessário, o desenvolvimento de mais estudos que ofereçam contribuições e evidências para uma melhor compreensão relativamente aos diferentes níveis de atividade física na função cognitiva e depressão em idosos. Diante deste contexto, o objetivo do presente estudo foi verificar o efeito dos diferentes níveis de atividade física na função cognitiva e depressão em idosos.

\section{MÉTODO}

O presente estudo pode ser caracterizado como observacional, de abordagem quantitativa, com delineamento longitudinal. Foi utilizada uma amostra de conveniência constituída por 465 idosos provenientes de uma amostra de 500 idosos $(69,93 \pm 6,63$ anos de idade), residentes em Salvador. Sendo 70,5\% de mulheres e 29,5\% de homens. Destes, 52\% não tinham cônjuge e 48\% eram casados (as). O maior percentual da renda mensal familiar foi para aqueles que possuíam rendimentos entre 1 e 4 salários mínimos1 (44,9\%), o menor foi para 1 salário mínimo (12,7\%), os demais rendimentos apresentaram frequência de 25,2\% para 5 a 7 salários mínimos e 17,2\% para aqueles que possuíam rendimentos maior que 8 salários mínimos. A maior parte da amostra $(44,5 \%)$ declarou ter nível secundário de escolaridade (até 11 anos de estudo formal), 17,4\% possuía nível superior (>11 anos de estudo

1 O valor de 1 salário mínimo no Brasil corresponde a aproximadamente 240 euros, conforme cotação atual do euro no Banco Central do Brasil (Brasil, 2017). 
formal) e 38,1\% declararam ter o nível básico de escolaridade (até 8 anos de estudo formal). Os idosos praticantes de diferentes programas de atividade física, com no mínimo um ano de prática, representaram $51,6 \%$ da amostra, enquanto os idosos não praticantes de nenhum tipo de programa de atividade física, com no mínimo um ano sem prática, representaram 48,4\% da amostra. Os idosos praticantes de atividade física participavam de programas que foram categorizados em 3 grupos: (i) grupo das danças (treino aeróbio, flexibilidade e equilibrio), 12,5\% da amostra; (ii) grupo de ginástica (treino de força, resistência, flexibilidade e equilibrio), 17,0\%; e (iii) grupo de prática dupla, relativo ao grupo de idosos que participavam de dois tipos de programas de atividade física (22,1\%). A frequência nestes programas de atividade física foi de 2 vezes por semana, com duração de 60 minutos por treino. Os participantes da amostra cumpriram um conjunto de critérios previamente estabelecidos para inclusão: ter idade igual ou superior a 60 anos, ter autonomia e independência na realização das suas atividades cotidianas. Como critério de exclusão, faltar a um dos momentos avaliativos na recolha dos dados ou apresentar incapacidades físicas ou mentais que o impossibilitasse na realização de atividade física regular, de tarefas do cotidiano ou no desempenho adequado da sua função cognitiva.

Para avaliação do nível de atividade física foi utilizado o International Physical Activity Questionnaire (IPAQ) versão longa (Matsudo et al., 2002; Craig et al., 2003; Mazo \& Benedetti, 2010), que permite estimar por autorrelato, o tempo semanal gasto em atividades físicas em diferentes contextos do cotidiano, categorizados em 4 domínios das atividades físicas: (i) trabalho; (ii) meio de transporte; (iii) tarefas domésticas; e (iv) tempo livre em recreação, esporte, exercícios e lazer. O IPAQ está validado e adaptado à população brasileira de adultos e idosos (Matsudo et al, 2002; Mazo \& Benedetti, 2010). Os dados foram calculados de acordo as orientações de processamento e de análise dos dados propostas por Matsudo et al (2002) e definidos os níveis de atividade física de acordo os seguintes critérios: nível ativo (muito ativo/ativo), aquele que cumpriu as recomendações de: a) Vigorosa: $\geq 3$ dias/sem e $\geq 20$ minutos por sessão; e/ou b) Moderada ou Caminhada: $\geq 5$ dias/ sem e $\geq 30$ minutos por sessão; e ou c). Qualquer atividade somada: $\geq 5$ dias/sem e $\geq 150$ minutos/sem (caminhada + moderada + vigorosa); Insuficientemente ativo: aquele que realiza atividade física, porém 
insuficiente para ser classificado como ativo, pois não cumpre as recomendações quanto à frequência ou duração. São divididos em "insuficientemente ativo A", aquele que atinge pelo menos um dos critérios da recomendação quanto à frequência ou quanto à duração da atividade: a) Frequência: 5 dias /semana ou b) Duração: 150 min / semana; e "insuficientemente ativo B", aquele que não atingiu nenhum dos critérios da recomendação quanto à frequência nem quanto à duração; Sedentário: aquele que não realizou nenhuma atividade física por pelo menos 10 minutos contínuos durante a semana.

A avaliação da função cognitiva foi realizada através do Mini Exame do Estado Mental (MEEM) (Bertolucci et al., 1994; Brucki et al., 2003) com o objetivo de verificar possíveis declínios da função cognitiva. O teste é composto por categorias que testam cinco aspectos do funcionamento cognitivo: orientação temporal e espacial; memória imediata e de evocação; atenção; cálculo; linguagem e capacidade construtiva visual. O MEEM foi desenvolvido por Folstein et al (1975), nomeadamente Mini Mental State Examination (MMSE) e posteriormente adaptado e validado para o Brasil por Bertolucci et al (1994) e Brucki et al (2003). Neste estudo foi utilizado o ponto de corte sugerido por Brucki et al (2003) para classificar os idosos com declínio cognitivo, de acordo com os níveis de escolaridade: 20 pontos para analfabetos; 25 pontos para idosos de 1 a 4 anos de estudo formal; 26,5 pontos para idosos de 5 a 8 anos de estudo formal; e 26 pontos para idosos que estudaram acima de 8 anos; 28 pontos para aqueles de 9 a 11 anos de estudo formal e 29 pontos para aqueles com mais de 11 anos de estudo formal.

A depressão nos idosos foi avaliada através do Beck Depression Inventory 2nd (BDI-II) com o objetivo de verificar a intensidade da depressão (Beck, Steer \& Carbin, 1988). A versão brasileira do BDI-II foi aprovada pelo Sistema de Avaliação de Testes Psicológicos (SATEPSI) do Consetho Federal de Psicologia do (CFP) do Brasil, com a devida autorização do The Psychological Corporation, em versão validada e atualizada por Gomes-Oliveira et al (2012). Os pontos de corte deste instrumento, segundo as recomendações de Gomes-Oliveira et al (2012) devem ser de acordo as características da amostra. Neste estudo, adotou-se os pontos de corte em conformidade com as normas da versão do instrumento em português para o Brasil, nas quais os níveis de intensidade da depressão estão subdivididos em: 0 a 11 (mínimos); de 11 a 19 (leve); 
de 20 a 35 (moderado) e de 36 a 63 (grave) (Cunha, 2001; Gorenstein, Pang, Argimon \& Werlang, 2012)

Os dados foram recolhidos na cidade de Salvador, após cumpridos os aspectos formais de pedidos de autorização às instituições selecionadas e apresentação do projeto da pesquisa, foi viabilizado o contato entre a investigadora e participantes. Os participantes concordaram em participar do presente estudo, em acordo com os procedimentos éticos definidos no Termo de Consentimento Livre e Esclarecido (TCLE). Os momentos da recolha dos dados ocorreram em 35 semanas, período formal da realização das atividades (físicas, sociais, culturais e educativas) nas instituições selecionadas. Realizado o $1^{\circ}$ momento avaliativo (avaliação inicial), após 15 semanas, realizou-se o $2^{\circ}$ momento avaliativo (avaliação intermédia), 20 semanas depois, foi realizado o $3^{\circ}$ momento avaliativo (avaliação final).

Os instrumentos utilizados no presente estudo foram aplicados por uma equipa constituída pela investigadora e colaboradores treinados para o efeito. Cada momento avaliativo teve a duração de 10 dias, nos quais os instrumentos foram administrados em grupos de aproximadamente 25 idosos. Devido a restrição de salas adequadas para a aplicação dos instrumentos, os grupos de idosos foram divididos nos turnos matutino e vespertino, com duração média de uma hora e meia por aplicação. A recolha dos dados ocorreu em locais previamente marcados, como sedes de associações de bairros, de centros sociais urbanos, igrejas e de uma universidade aberta a terceira idade.

Esta pesquisa foi submetida ao julgamento da Comissão de Ética da Universidade de Trás-os-Montes e Alto Douro (UTAD), Portugal, sendo aprovado com protocolo n 11/2015 e adotou os princípios éticos dispostos na Resolução do Conselho Nacional de Saúde (n 196/96) do Brasil.

Para a construção da base de dados e posteriores análises estatísticas, utilizou-se a versão 22.0 do SPSS® (Statistical Package for Social Sciences). Foi aplicado o modelo geral linear (GLM), e utilizada uma MANCOVA de medidas repetidas após a avaliação inicial dos exercícios físicos, cruzando os três momentos (avaliação inicial; avaliação intermédia e avaliação final), de forma a verificar o efeito entre as variáveis independentes (diferentes níveis de atividades físicas) e variáveis dependentes (função cognitiva e depressão) através das diferenças dos grupos, 
introduzindo no modelo a idade, como covariável. Admitiu-se o nível de significância de 5\% (ps0.05).

\section{RESULTADOS}

Para verificar a frequência dos níveis de atividade física nos 3 momentos avaliativos, os participantes foram divididos em 4 grupos (ativo, insuficientemente ativo $A$, insuficientemente ativo B e sedentário), em acordo os critérios de classificação dos níveis de atividade física definidos no IPAQ (Matsudo et al, 2002;Mazo \& Benedetti, 2010). A tabela 1 mostra a frequência por nível de atividade física nos 3 momentos avaliativos:

Tabela 1 - Frequência por nível de atividade físicas (AF) na avaliação inicial, avaliação intermédia e avaliação final

\begin{tabular}{|c|c|c|c|c|c|c|}
\hline Níveis AF & \multicolumn{2}{|c|}{ Av. Inicial } & \multicolumn{2}{c|}{ Av. Intermédia } & \multicolumn{2}{c|}{ Av. Final } \\
\hline \multirow{2}{*}{ Ativo } & $\mathrm{N}$ & $\%$ & $\mathrm{~N}$ & $\%$ & $\mathrm{~N}$ & $\%$ \\
& 93 & 20 & 263 & 56,6 & 261 & 56,1 \\
Insuf ativo A & 98 & 21,1 & 39 & 8,4 & 32 & 6,9 \\
Insuf ativo B & 154 & 33,1 & 57 & 12,3 & 65 & 14,0 \\
Sedentário & 120 & 25,8 & 106 & 22,8 & 107 & 23,0 \\
Total & 465 & 100,0 & 465 & 100,0 & 465 & 100,0 \\
\hline
\end{tabular}

Os resultados da tabela 1 demonstram as seguintes frequências: idosos ativos (avaliação inicial: 20\%; avaliação intermédia: 56,6\% e avaliação final: 56,1\%), idosos insuficientemente ativo A (avaliação inicial: 21,1\%; avaliação intermédia: 8,4\% e avaliação final: 6,9\%); idosos insuficientemente ativos B (avaliação inicial: 33,1\%; avaliação intermédia: 12,3\% e avaliação final: 14,0\%), por fim os idosos sedentários (avaliação inicial: 25,8\%; avaliação intermédia: 22,8\% e avaliação final: 23,0\%). Observou-se um aumento relevante da frequência de idosos ativos ao longo do estudo, assim como a redução de idosos insuficientemente ativos A e B, e uma estabilidade percentual de idosos sedentários.

A tabela 2 apresenta as médias e desvio padrão da função cognitiva 
(MEEM) associadas aos níveis de atividade física na avaliação inicial, avaliação intermédia e avaliação final.

Tabela 2 - Médias e desvio padrão - Função cognitiva (MEEM) e níveis de atividade física

\begin{tabular}{|c|c|c|c|c|c|c|c|c|c|}
\hline \multirow{2}{*}{$\begin{array}{c}\text { Níveis de } \\
\text { Atividade Física }\end{array}$} & \multicolumn{3}{|c|}{ Av. Inicial } & \multicolumn{3}{|c|}{ Av. Intermédia } & \multicolumn{3}{|c|}{ Avaliação final } \\
\hline & $N$ & Média $\pm D P$ & IC95\% & $\mathrm{N}$ & Média \pm DP & IC95\% & $N$ & Média $\pm D P$ & IC95\% \\
\hline Ativo & 93 & $29,20 \pm 2,38$ & $28,71-29,69$ & 263 & $29,84 \pm 1,88$ & $29,04-29,98$ & 261 & $29,83 \pm 1,82$ & $\begin{array}{l}29,05- \\
29,93\end{array}$ \\
\hline Ins.ativo A & 98 & $29,18 \pm 2,52$ & $28,68-29,70$ & 39 & $27,51 \pm 4,60$ & $26,38-28,65$ & 32 & $27,31 \pm 5,10$ & $\begin{array}{l}26,78- \\
28,90\end{array}$ \\
\hline Ins. ativo $B$ & 154 & $28,69 \pm 3,25$ & $28,17-29,12$ & 57 & $25,70 \pm 5,20$ & $24,77-26,65$ & 65 & $25,77 \pm 5,22$ & $\begin{array}{l}24,86- \\
26,72\end{array}$ \\
\hline Sedentários & 120 & $26,75 \pm 3,53$ & $25,91-27,59$ & 106 & $25,42 \pm 5,15$ & $24,72-26,10$ & 107 & $24,61 \pm 5,52$ & $\begin{array}{c}24,12- \\
26,17\end{array}$ \\
\hline Total & 465 & $28,40 \pm 3,53$ & $28,07-28,72$ & 465 & $28,13 \pm 4,13$ & $28,08-28,77$ & 465 & $27,89 \pm 4,42$ & $\begin{array}{l}26,45- \\
28,02\end{array}$ \\
\hline
\end{tabular}

Os resultados da tabela 2 demonstram que no teste cognitivo (MEEM), os valores mais elevados foram obtidos pelos idosos ativos nos 3

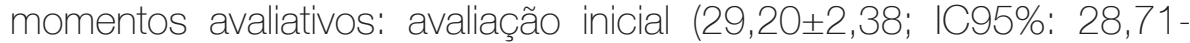

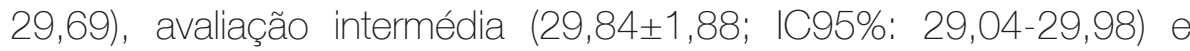

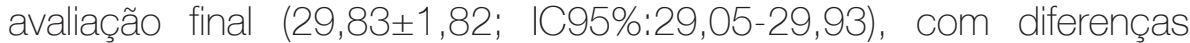
significativas ( $\mathrm{p}=0.000)$.

A tabela 3 apresenta as médias e desvio padrão da depressão de acordo com os níveis de atividade física na avaliação inicial, avaliação intermédia e avaliação final.

Tabela 3 - Médias e desvio padrão - Depressão (BDI-II) e níveis de atividade físicas

\begin{tabular}{|c|c|c|c|c|c|c|c|c|c|c|}
\hline \multirow{2}{*}{$\begin{array}{l}\text { Níveis de } \\
\text { atividade } \\
\text { física }\end{array}$} & \multicolumn{3}{|c|}{ Avaliação inicial } & \multicolumn{3}{|c|}{ Avaliação intermédia } & \multicolumn{4}{|c|}{ Avaliação final } \\
\hline & $\mathrm{N}$ & Média \pm DP & IC95\% & $\mathrm{N}$ & Média \pm DP & IC95\% & $\mathrm{N}$ & Média \pm DP & IC95\% & $p^{*}$ \\
\hline Ativo & 93 & $12,34 \pm 6,82$ & $10,94-13,75$ & 263 & $9,65 \pm 0,451$ & $8,77-10,53$ & 261 & $8,35 \pm 5,47$ & $7,65-10,65$ & 0.000 \\
\hline Ins.ativo A & 98 & $12,03 \pm 7,27$ & $10,57-13,49$ & 39 & $15,67 \pm 1,17$ & $13,37-17,97$ & 732 & $14,84 \pm 8,77$ & $13,68-15,75$ & 50.000 \\
\hline Ins. ativo $B$ & 154 & $12,40 \pm 7,02$ & $11,28-13,52$ & 57 & $17,18 \pm 0,97$ & $15,27-19,07$ & 765 & $18,00 \pm 9,52$ & $15,45-19,20$ & 00.001 \\
\hline Sedentários & 120 & $15,70 \pm 9,25$ & $14,03-17,37$ & 106 & $18,15 \pm 0,71$ & $16,76-19,54$ & 4107 & $19,02 \pm 9,08$ & $18,76-19,78$ & 80.001 \\
\hline Total & 465 & $13,16 \pm 7,80$ & $12,45-13,87$ & 465 & $13,0 \pm 8,26$ & $12,54-13,43$ & 3465 & $12,66 \pm 8,83$ & $11,39-13,53$ & 30.000 \\
\hline
\end{tabular}

Os resultados na tabela 3 indicam que os idosos ativos apresentaram os menores valores para depressão na avaliação inicial (12,34t6,82;

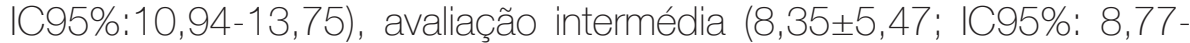

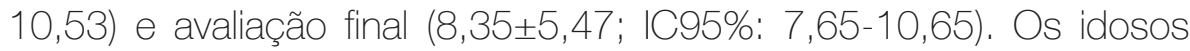
sedentários apresentaram os maiores valores no BDI-\|l na avaliação 


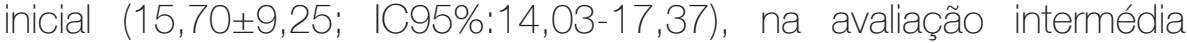

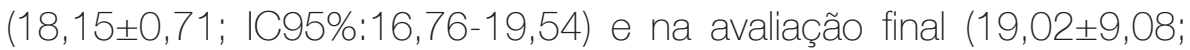
IC95\%:18,76-19,78). O nível ativo apresentou diferença significativa para todos os demais níveis ( $\mathrm{p}=0.000)$. O nível insuficientemente ativo A evidenciou diferença significativa para o nível ativo ( $\mathrm{p}=0.000)$. Já o nível insuficientemente ativo B evidenciou diferença significativa apenas para o nível ativo ( $\mathrm{p}=0.001)$ e foram encontradas diferenças significativas dos sedentários para o ativo ( $\mathrm{p}=0.001)$.

Relativamente aos efeitos principais entre os níveis de atividade física e a função cognitiva, a tabela 4 apresenta os resultados obtidos na MANCOVA de medidas repetidas.

Tabela 4 - Efeitos principais das variáveis independentes no MEEM

\begin{tabular}{|c|c|c|c|c|c|c|c|c|c|}
\hline \multirow{2}{*}{$\begin{array}{l}\text { Efeitos } \\
\text { principais }\end{array}$} & \multicolumn{9}{|c|}{ Função cognitiva (MEEM) } \\
\hline & \multicolumn{3}{|c|}{ Avaliação inicial } & \multicolumn{3}{|c|}{ Avaliação intermédia } & \multicolumn{3}{|c|}{ Avaliação final } \\
\hline \multirow{2}{*}{$\begin{array}{l}\text { Níveis de } \\
\text { atividade física }\end{array}$} & $F$ & Sig. & $\eta^{2}$ & $F$ & Sig. & $\eta^{2}$ & $F$ & Sig. & $\eta^{2}$ \\
\hline & 2,067 & 0,101 & 0,014 & 3,116 & 0,026 & 0,021 & 4,673 & 0,003 & 0,031 \\
\hline
\end{tabular}

Os resultados evidenciaram que os níveis de atividade física não apresentaram efeito significativo na função cognitiva na avaliação inicial ( $F=$ 2,067; Wilks' Lambda=0,993, $\mathrm{p}=0,101$; h2=0,014), mas na avaliação intermédia foi verificado um efeito significativo ( $F=3,116, p=0.000$, Wilks' Lambda=0,989, h2=0,026), assim como na avaliação final também foi verificado efeito significativo ( $F=4,673$, Willks' Lambda= $0,981, p=0,003 ; h 2=0,031)$.

A tabela 5 apresenta os efeitos principais entre os níveis de atividade física e a depressão, respectivamente, obtidos na MANCOVA de medidas repetidas.

Tabela 5 - Efeitos principais das variáveis independentes no BDI-II

\begin{tabular}{|llllllllll|}
\hline \multirow{2}{*}{$\begin{array}{l}\text { Efeitos } \\
\text { principais }\end{array}$} & \multicolumn{8}{c|}{ Depressão (BDI-II) } \\
\cline { 2 - 10 } & Avaliação inicial & \multicolumn{7}{c|}{ Avaliação intermédia } & Avaliação final \\
\hline $\begin{array}{l}\text { Níveis de } \\
\text { atividade } \\
\text { física }\end{array}$ & $F$ & Sig. & $\eta^{2}$ & $F$ & Sig. & $\eta^{2}$ & $F$ & Sig. & $\eta^{2}$ \\
\cline { 2 - 10 } & 2,737 & 0,043 & 0,019 & 4,170 & 0,006 & 0,028 & 2,725 & 0,029 & 0,025 \\
\hline
\end{tabular}

Na tabela 2, os resultados indicam que na depressão, foi evidenciado 
um efeito significativo na avaliação inicial ( $F=2,737$, Wilks' Lambda= 0,946, $p=0,043, h 2=0,019)$, na avaliação intermédia ( $F=4,170$, Wilks' Lambda $=0,989, h 1=0,028)$ e na avaliação final $(F=2,725$, Wilks' Lambda $=0,980, p=0,029, \mathrm{~h} 2=0,025)$.

\section{DISCUSSÃO}

O objetivo do presente estudo foi verificar o efeito dos diferentes níveis de atividade física na função cognitiva e na depressão de idosos. Os resultados indicaram que houve um efeito significativo do nível de atividade física na função cognitiva a partir da avaliação intermédia, e também na depressão nos três momentos avaliativos.

Esses resultados corroboram com muitos estudos encontrados na literatura, os quais indicam o efeito significativo dos níveis de atividade física na função cognitiva (Pereira et al., 2007; Erickson et al, 2011; Maass et al, 2016) e na depressão em idosos (Blumenthal et al, 2012; Lindwall et al, 2011; Paranthaman et al, 2012; Carneiro et al, 2015).

O efeito da atividade física nos processos neurofisiológicos envolvidos nos mecanismos subjacentes ao desempenho da função cognitiva, tem demonstrado o aumento da liberação e da síntese de diversos fatores neurotróficos relacionados à neurogênese adulta, plasticidade cerebral (Pereira et al, 2007; Erickson et al, 2011) e também à angiogênese (Sonntag et al, 2007).

Esse efeito pode favorecer a preservação ou aumento da reserva cognitiva no envelhecimento (Nithianantharajah \& Hannan, 2009), já que, de acordo com Lee, Clemenson e Gage (2012), as taxas de neurogênese e o volume do hipocampo diminuem intensamente com o avançar da idade, aumentando as chances do desenvolvimento do declínio cognitivo e ou demências.

Entretanto, o efeito da atividade física pode aumentar de modo significativo o fluxo sanguíneo no giro denteado, aumentar o volume do hipocampo e consequentemente a neurogênese nesta área (Erickson et al, 2011), e ainda preservar ou aumentar a reserva cognitiva no envelhecimento (Nithianantharajah \& Hannan, 2009), sendo correlacionado ao aumento dos níveis de BDNF (Erickson et al, 2011), da neuroplasticidade sináptica e de fatores de neuroproteção ao declínio cognitivo e 
demências (Pereira et al, 2007).

Constata-se, portanto, que muitos estudos indicam evidências de que a prática de atividade física no envelhecimento é benéfica ao desempenho satisfatório da função cognitiva, para prevenir e ou protelar o declínio cognitivo ou demência, assim como a depressão. No presente estudo, os idosos ativos apresentaram ao longo do estudo os melhores valores nas avaliações da função cognitiva e da depressão, comparados aos idosos com níveis inferiores de atividade física.

$\mathrm{Na}$ intensidade da depressão, nossos resultados sugeriram um efeito benéfico da atividade física, uma vez que os idosos ativos apresentaram os menores valores no BDI-Il ao longo do estudo, ao contrário dos idosos com os níveis mais baixos de atividade física (insuficiente ativo A, B e sedentários). Com resultados semelhantes, Motl et al (2005) indicaram que idosos ativos apresentaram uma redução significativa nos níveis de depressão em um estudo longitudinal, em oposto aos idosos sedentários.

Estudos sugerem que o efeito do nível ativo de atividade física pode beneficiar mecanismos neurofisiológicos do sistema límbico-cortical-estriatal-pálido-talâmico (Lacerda et al, 2004), melhorar a regulação da atividade do eixo hipotálamo-pituitária-adrenal (HPA) associada ao 5H-T (5-hidroxitriptamina), sistema de serotoninas (Archer et al, 2015), favorecer o aumento dos níveis de VEGF e a angiogênese, (Sonntag, et al, 2007) e ainda regular os níveis do IGF-1 (Maass et al., 2016), cujos níveis muito elevados foram associados aos sintomas depressivos (Bot et al, 2016) e os reduzidos foram associados ao declínio cognitivo (Maass et al, 2016).

No entanto, Dunn, Trivedi, Kampert, Clark e Chambliss (2005) não encontraram efeito significativo do exercício físico em indivíduos com depressão grave em relação ao grupo que não realizou exercícios. Os autores concluíram que o efeito foi eficaz para indivíduos com depressão leve e moderada. Lindwall et al (2011), no entanto, destacaram o efeito benéfico da atividade física regular nos sintomas depressivos, mas ressaltaram que os indivíduos com depressão grave, devido à natureza dos sintomas, não se envolvem em um programa de treinamento físico. Blumenthal et al (2012) em ampla revisão da literatura, verificaram que o exercício físico pode ser um tratamento eficaz para a depressão, independente da sua intensidade, em medida comparável ao trata- 
mento farmacológico e à psicoterapia. Os autores afirmam que estudos observacionais sugerem que indivíduos ativos são menos propensos a desenvolver depressão, e os estudos de intervenção sugerem que o efeito do nível ativo de atividade física é benéfico na redução dos níveis de depressão.

Neste sentido, a prática regular de atividade física tem se mostrado eficaz para prevenir ou atenuar a depressão, também considerada um dos fatores de risco para o declínio cognitivo e consequente desenvolvimento de demência (Baer, Tabri, Blair, Bye, Li \& Pushkar, 2013). Esses agravos, sejam separados ou associados, podem trazer sérias limitações à realização de tarefas cotidianas, independência, autonomia e capacidade funcional do idoso, com um possível aumento do isolamento social, estado de solidão e da demanda por cuidados de familiares, amigos e serviços de saúde (Faber, Scheicher, \& Soares, 2017). Percebe-se, portanto, que a depressão ou o declínio cognitivo em associação aos demais efeitos deletérios do envelhecimento podem agravar o estado de saúde geral do idoso (Paranthaman et al, 2012), a depressão no nível leve, pode evoluir para moderada ou grave (Beck et al, 1988) ou o declínio cognitivo progredir para demência (Baer et al., 2013). No entanto, muitos estudos sugerem que a atividade física regular demonstra um efeito benéfico tanto para a redução dos níveis de depressão (Blumenthal et al, 2012; Lindwall et al, 2011; Paranthaman et al, 2012; Carneiro et al, 2015), quanto para a prevenção do declínio cognitivo (Pereira et al., 2007; Erickson et al, 2011; Maass et al, 2016). Outros estudos apontam a prática regular de atividade física, como fator protetor à depressão (Du et al, 2015), de modo inverso, o comportamento sedentário tem sido considerado fator de risco (Gudmundsson et al, 2015).

Não podemos, contudo, generalizar nossos resultados, visto que foram utilizados instrumentos de medidas por autorrelato, o IPAQ, MEEM e o BDI-II. Este facto poderá ser visto como uma limitação do nosso estudo, embora sejam instrumentos utilizados em inúmeros estudos longitudinais em vários países, com comprovada validade e fidedignidade, mas por se tratar de instrumentos de autorrelato estão propensos ao viés nas informações. Sugere-se, então, que em estudos futuros, associados ao IPAQ sejam utilizados instrumentos de medidas mais precisos, como o pedômetro ou acelerômetro. Para a verificação do desempenho da 
função cognitiva, sugere-se que o MEEM seja um dos instrumentos inseridos em uma avaliação neuropsicológica mais ampla e aprofundada. De modo semelhante, sugerimos que o BDI-II seja inserido em avaliações clínicas realizadas por psiquiatras ou psicólogos.

Os horários de aplicação dos instrumentos (em turnos matutino e vespertino), também pode ser considerada outra limitação do nosso estudo, ao considerar que o estado emocional ou desempenho cognitivo dos indivíduos podem variar ao longo do dia, sugere-se, então para estudos futuros uma uniformização do horário de aplicação dos instrumentos. Sugerimos também que em investigações futuras, o efeito dos níveis de atividade física na função cognitiva e depressão de idosos, sejam verificados para além dos aspectos gerais, nos aspectos específicos da função cognitiva (memória de trabalho, raciocínio lógico, funções executivas, entre outros) e da depressão (aspectos cognitivos, afetivos e somáticos).

\section{CONCLUSÃO}

Diante das evidências encontradas no presente estudo, conclui-se que a atividade física demonstrou um efeito benéfico à função cognitiva e para redução da depressão nos idosos da amostra estudada. Os idosos ativos apresentaram um desempenho satisfatório na função cognitiva e reduzidos níveis de depressão, ao contrário dos idosos com baixos níveis de atividade física. Sendo assim, destacamos a importância dos resultados obtidos no nosso estudo para a busca de estratégias de promoção à saúde dos idosos através de atividades físicas regulares que visem a mauntenção da independência, autonomia e capacidade funcional do idoso e que possam prevenir ou atenuar a depressão e o declinio da função cognitiva.

\section{BIBLIOGRAFIA}

Archer, T., Josefsson, T., \& Lindwall, M. (2015). Effects of Physical Exercise on Depressive Symptoms and Biomarkers in Depression. CNS \& Neurological Disorders - Drug Targets, 13(10), 1640-1653.

Baer, L. H., Tabri, N., Blair, M., Bye, D., Li, K. Z., \& Pushkar, D. (2013). Lon- 


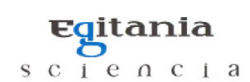

gitudinal associations of need for cognition, cognitive activity, and depressive symptomatology with cognitive function in recent retirees. The Journals of Gerontology: Series B, 68(5), 655-664.

Beck, A., Steer, R., \& Carbin, M. (1988). Psychometric properties of the Beck Depression Inventory: Twenty-five years of evaluation. Clinical Psychology Review, 8(1), 77-100.

Brasil (2017). Banco Central do Brasil. Conversão de moedas. http://www4.bcb. gov.br/pec/conversao/conversao.asp.

Benedetti, T., Borges, L., Petroski, E., \& Gonçalves, L. (2008). Atividade física e estado de saúde mental de idosos. Rev de Saú Púb, 42(2), 302-307.

Bertolucci, P.H.F.; Brucki, S.M.D.; Campacci, S.R. \& Juliano, Y (1994). O Mini-Exame do Estado Mental em uma população geral: impacto da escolaridade. Arq. Neuro-Psiquiatr, 52(1), 01-07.

Blumenthal, J. A., Smith, P. J., \& Hoffman, B. M. (2012). Is Exercise a Viable Treatment for Depression? ACSM's Health \& Fitness Journal, $16(4), 14$.

Bot, M., Milaneschi, Y., Penninx, B., \& Drent, M. (2016). Plasma insulin-like growth factor I levels are higher in depressive and anxiety disorders, but lower in antidepressant medication users. Psychoneuroendocrinology, 68, 148-155.

Brucki S.M.D., Nitrini R., Caramelli P., Bertolucci P.H.F. \& Okamoto, I. H. (2003) Sugestões para o uso do mini-exame do estado mental no Brasil. Arq Neuropsiquiatr., $61(3 B), 777-781$.

Carneiro, L. S. F., Fonseca, A. M., Vieira-Coelho, M. A., Mota, M. P., \& Vasconcelos-Raposo, J. (2015). Effects of structured exercise and pharmacotherapy vs. pharmacotherapy for adults with depressive symptoms: A randomized clinical trial. Jour of Psych Res, 71 (July), 48-55.

Colcombe, S., \& Kramer, A. F. (2003). Fitness Effects on the Cognitive Function of Older Adults: A Meta-Analytic Study. Psychological Science, 14(2), $125-130$. Chang, Y.K., Labban, J.D., Gapin, J.I., \& Etnier, J.L. (2012). The effects of acute exercise on cognitive performance: a meta-analysis. Brain Research, 1453, 87101. PubMed.

Craig, Cora L., Marshall, Alison L., Sjorstrom, Michael, Bauman, Adrian E., Booth, Michael L., Ainsworth, Barbara E., Pratt, Michael, Ekelund, Ulf, Yngve, Agneta, Sallis, James .F and Oja, Pekka (2003) International physical activity questionnaire: 12-country reliability and validity. Medicine and Science in Sports and Exercise, 35 8: 1381-1395.

Cunha, J. A. (2001). Manual da Versão em Português das Escalas Beck. São Paulo: Casa do Psicólogo.

Deslandes, A. (2013). The biological clock keeps ticking, but exercise may turn it back. Arq Neuropsiquiatr., 71, $113-118$.

Du, W. J., Tan, J.P., Yi, F., Zou, Y.M., Gao, Y., Zhao, Y.M., \& Wang, L.N. (2015). Physical activity as a protective factor against depressive symptoms in older Chi- 


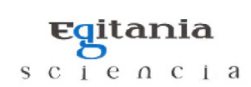

nese veterans in the community: result from a national cross-sectional study. Neuropsychiatric Disease and Treatment, 11, 803-813.

Dunn, A., Trivedi, M., Kampert, J., Clark, C. \& Chambliss, H. (2005). Exercise treatment for depression. American Journal of Preventive Medicine, 28(1), 1-8.

Erickson, K.I., Voss, M.W., Prakash, R.S., Basak, C., Szabo, A., Chaddock, L., ... Kramer, A.F. (2011). Exercise training increases size of hippocampus and improves memory. Proc Natl Acad Sci USA, 108(7), 3017-3022.

Faber, L. M., Scheicher, M. E., \& Soares, E. (2017). Depressão, Declínio Cognitivo e Polimedicação em idosos institucionalizados. Revista Kairós Gerontologia, 20(2), 195-210. ISSNe 2176-901X. São Paulo (SP), Brasil: FACHS/NEPE/ PEPGG/PUC-SP

Folstein M.F., Folstein S.E., \& McHugh P.R. (1975). Mini-mental state: a practical method for grading the cognitive state of patients for the clinician. J Psychiatric Res, 12 (3), 189-98.

Frazer, C. J., Christensen, H., \& Griffiths, K. M. (2005). Effectiveness of treatments for depression in older people. Medical Journal of Australia, 182(12), 627-32. Gomes-Oliveira, M., Gorenstein, C., Neto, F., Andrade, L., \& Wang, Y. (2012). Validation of the Brazilian Portuguese Version of the Beck Depression Inventory-ll in a community sample. Rev. Bras. de Psiq., 34 (4), 389-394.

Gorenstein, C., Pang, W. Y., Argimon, I. L., \& Werlang, B. S. G. (2012). BDIIl Manual do Inventário de Depressão de Beck. São Paulo: Casa do Psicólogo Livraria e Editora Ltda.

Gudmundsson, P., Lindwall, M., Gustafson, D. R., Östling, S., Hällström, T., Waern, M., \& Skoog, I. (2015). Longitudinal associations between physical activity and depression scores in Swedish women followed 31 years. Acta Psychiatrica Scandinavica, 132(6), 451-458. http://doi.org/10.1111/acps.12419.

Hillman, C.H., Erickson, K.I., \& Kramer, A.F. (2008). Be smart, exercise your heart: exercise effects on brain and cognition. Nature Reviews. Neuroscience, 9, 58-65. PubMed.

Hötting, K., \& Röder, B. (2013). Beneficial effects of physical exercise on neuroplasticity and cognition. Neuroscience \& Biobehavioral Reviews, 37(9, Part B), 2243-2257.

Lacerda, A., Keshavan, M., Hardan, A., Yorbik, O., Brambilla, P., Sassi, R., ... Soares, J. C. (2004). Anatomic evaluation of the orbitofrontal cortex in major depressive disorder. Biological Psychiatry, 55(4), 353-358.

Lee, S., Clemenson, G., \& Gage, F. (2012). New neurons in an aged brain. Behavioural Brain Research, $227(2), 497-507$.

Lindwall, M., Larsman, P., \& Hagger, M. (2011). The reciprocal relationship between physical activity and depression in older European adults: A prospective cross-lagged panel design using SHARE data. Health Psychology, 30(4), 453- 
462.

Krishnan, V., \& Nestler, E. (2008). The molecular neurobiology of depression. Nature, 455(7215), 894-902.

Maass, A., Düzel, S., Brigadski, T., Goerke, M., Becke, A., Sobieray, U., ... Düzel, E. (2016). Relationships of peripheral IGF-1, VEGF and BDNF levels to exercise-related changes in memory, hippocampal perfusion and volumes in older adults. Neuroimage, 131, 142-154.

Matsudo, S., Matsudo, V., Araújo, T., Andrade, D., Andrade, E., Oliveira, L.C., \& Braggion G. (2002). Nível de atividade física da população do Estado de São Paulo: análise de acordo com o gênero, idade, nível socioeconômico, distribuição geográfica e de conhecimento. Rev. Bras. Ciên. e Mov., 10(4), 41-50.

Mazo, G. \& Benedetti, T. (2010). Adaptação do questionário internacional de atividade física para idosos. Rev. Bras. de Cineantrop. \& Des. Hum., 11 (6), 480 484.

Motl, R., Konopack, J., McAuley, E., Elavsky, S., Jerome, G., \& Marquez, D. (2005). Depressive Symptoms Among Older Adults: Long-Term Reduction After a Physical Activity Intervention. J Behav Med, 28(4), 385-394.

Nithianantharajah, J., \& Hannan, A. J. (2009). The neurobiology of brain and cognitive reserve: Mental and physical activity as modulators of brain disorders. Progress in Neurobiology, 89(4), 369-382.

Paranthaman, R., Greenstein, A., Burns, A., Heagerty, A., Malik, R., \& Baldwin, R. (2012). Relationship of endothelial function and atherosclerosis to treatment response in late-life depression. Int J Geriatr Psychiatry, 27(9), 967-973.

Pereira, A., Huddleston, D., Brickman, A., Sosunov, A., Hen, R., \& McKhann, G., ... Small, S. A. (2007). An in vivo correlate of exercise-induced neurogenesis in the adult dentate gyrus. Proceedings Of The National Academy Of Sciences, 104(13), 5638-5643.

Sonntag, W.E., Eckman, D.M., Ingraham, J. \& Riddle, D. R. (2007). Regulation of Cerebrovascular Aging. In: Riddle, D.R. (ed.). Brain Aging: Models, Methods, and Mechanisms. Boca Raton: CRC Press/Taylor \& Francis.

Underwood, M., Lamb, S. E., Eldridge, S., Sheehan, B., Slowther, A. M., Spencer, A., ... Taylor, S. J. C. (2013). Exercise for depression in elderly residents of care homes: a cluster-randomised controlled trial. The Lancet, 382(9886), 41-49. 